\title{
Oil-Spills Detection in Net-Sar Radar Images Using Support Vector Machine
}

\author{
Dong Zhi-Ming ${ }^{1, *}$, Guo Li-Xia ${ }^{1}$, Zeng Jian-Kui ${ }^{1}$ and Zhou Xue-Bin ${ }^{2}$

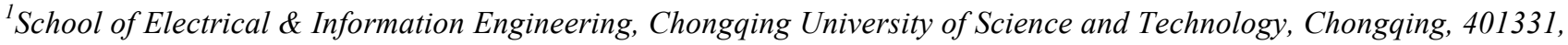 \\ P.R. China \\ ${ }^{2}$ Southwest Technology and Engineering Research Institute, Chongqing 400039, P.R. China
}

\begin{abstract}
Oil-spills detection is an important problem in many applications such as communication and navigation. Many methods have been presented for this problem. The Maximum Likelihood (ML) is one of the good solutions. But, in traditional algorithms for ML nonetheless, the computational load is very heavy and multivariate nonlinear maximization problem is serious. To deal with these problems, this paper describes an application of neural network (NN) for obtaining the global optimal solution of ML DOA estimation. It overcomes the local optima problem existing in some ML DOA estimation algorithms and improves the estimation accuracy. The computation complexity is modest.
\end{abstract}

Keywords: Artificial Neural Network (ANN), DOA estimation, Maximum likelihood.

\section{INTRODUCTION}

In many applications such as communication and navigation, the estimation of target direction-of-arrival (DOA) is one of the important problems. Lots of techniques for this problem have been proposed over the past decades. The Maximum Likelihood technique is one of the first to be investigated and best in theory [1]. Nonetheless, because of the high computational load of the multivariate nonlinear maximization problem involved, it could not become popular. Instead, suboptimal method with reduced computational load has governed the field. The better known ones are the MUSIC method of Schmidt [2], and the minimum norm method of Reddi [3] and Kumaresan and Tufts [4].

However, the ML method over-performs other methods in many aspects [5], especially, when the target echo is very small, or when the noise or clutter is very strong. In fact, many techniques cannot deal with the circumstances of coherent signals.

Many researchers have proposed various algorithms to maximize the likelihood function, wanting to guarantee global convergence within less computing time. Alternating projection method [6], simulated annealing algorithm [7], grid search approach, data-supported grid search [8], can approximately obtain the ML estimation. But most of them cannot guarantee global convergence in general case [9].

In this paper, a global optimization of neural network [10] is developed to search for the nonlinear global optimization solution of the maximum likelihood. And then, we study the performance of $\mathrm{NN}$ algorithm.

\footnotetext{
*Address correspondence to this author at the School of Electrical \& Information Engineering, Chongqing University of Science and Technology, Chongqing, 401331, P.R China; Tel: 023-65023987; E-mail: zhmdong@cqust.edu.cn
}

This paper is organized as follows. Section 2 presents the problem of target direction-of-arrival estimation. In section 3 , we propose the general neural network algorithm. And then, in section 4 the $\mathrm{NN}$ algorithm is applied to DOA estimation problem. Section 5 gives some simulation to verify the efficiency of our algorithm. Section 6 gives a conclusion to the whole paper.

\section{PROBLEM FORMULATION}

Consider an antenna array which is constituted of $\mathrm{M}$ sensors with arbitrary locations and arbitrary directional characteristics.

It is assumed that $\mathrm{L}$ narrow-band plane waves arrive on the antenna array from $\mathrm{L}$ locations $\theta_{1}, \theta_{2}, \ldots, \theta_{\mathrm{L}}$.

In array signal processing techniques, narrow-band in the sensor array context means that the propagation delays of the signals across the array are much smaller than the reciprocal of the bandwidth of the signals. So, it follows that the complex envelopes of the signals received by the array can be expressed as:

$$
X(\mathrm{t})=\sum_{K=1}^{L} a\left(\theta_{k}\right) s_{k}(\mathrm{t})+n(\mathrm{t})
$$

where

Where $\mathrm{X}(\mathrm{t})$ is the $\mathrm{M} \times 1$ vector

$$
X(\mathrm{t})=\left[\mathrm{x}_{1}(\mathrm{t}), \mathrm{x}_{2}(\mathrm{t}), \cdots, \mathrm{x}_{M}(\mathrm{t})\right]^{T}
$$

In formula (2), $\mathrm{T}$ denotes the transpose. And $\mathrm{a}\left(\theta_{\mathrm{k}}\right)$ is the steering vector of the array toward direction $\theta_{\mathrm{k}}$.

$$
a\left(\theta_{k}\right)=\left[a_{1}\left(\theta_{k}\right) \mathrm{e}^{-j \omega_{0} \tau_{1} \theta_{k}}, \cdots, a_{M}\left(\theta_{k}\right) \mathrm{e}^{-j \omega_{0} \tau_{M} \theta_{k}}\right]^{T}
$$


$n(\mathrm{t})=\left[n_{1}(\mathrm{t}), n_{2}(\mathrm{t}), \cdots, n_{M}(\mathrm{t})\right]^{T}$

Here:

$x_{i}(t)=$ the signal received by the ith sensor .

$s_{k}(t)=$ the signal emitted by the kth source.

$a_{i}\left(\theta_{k}\right)=$ the amplitude response of the ith sensor to a wave-front impinging from location $\theta_{k}$

$n_{i}(t)=$ the noise at the ith sensor.

Then, consider the clutter signal, it is similar to target echo. But it is a random signal which can be described by a coefficient $\zeta_{J k}$.

$C(\mathrm{t})=\sum_{K=1}^{L} \zeta_{J k} \mathrm{a}\left(\theta_{k}\right) s$

So, the signal received can be expressed as:

$X(\mathrm{t})=\sum_{K=1}^{L} a\left(\theta_{k}\right) s_{k}(\mathrm{t})+C(\mathrm{t})+n(\mathrm{t})$

The vector of the received signals $x(t)$ can be expressed more compactly as:

$X(\mathrm{t})=A(\Theta) s(\mathrm{t})+n(\mathrm{t})$

Where $\mathrm{A}(\Theta)$ is the $\mathrm{M} \times \mathrm{L}$ matrix of the steering vectors $A(\Theta)=\left[a\left(\theta_{1}\right), \ldots, a\left(\theta_{L}\right)\right]$. And $s(t)$ is $\mathrm{L} \times 1$ vector of the signals which can be expressed as $s(t)=\left[s_{I}(t), \ldots, s_{L}(t)\right]$.

The localization of target problem is to estimate the locations $\theta_{l}, \theta_{2}, \ldots, \theta_{L}$ of the sources from $\mathrm{N}$ target signal samples ("snapshots") of the received signals. The maximum likelihood estimation of the source localization problem is derived as[6].

$$
\begin{aligned}
\hat{\Theta} & =\arg \left\{\max _{\Theta} L(\Theta)\right\} \\
& =\arg \left\{\max _{\Theta} \operatorname{tr}\left(\mathrm{P}_{\mathrm{A}(\Theta)} \mathrm{R}\right)\right\}
\end{aligned}
$$

Where $\operatorname{tr}[\mathrm{C}$ is the trace of the bracketed matrix, $\mathrm{P}_{\mathrm{A}(\Theta)}=\mathrm{A}(\Theta)\left(\mathrm{A}^{\mathrm{H}}(\Theta) \mathrm{A}(\Theta)\right)^{-1} \mathrm{~A}^{\mathrm{H}}(\Theta)$ is the projection operator onto the space spanned by the columns of the matrix $A(\Theta)$.

Where, $R=\frac{1}{N} \sum_{i=1}^{N} \mathrm{X}\left(\mathrm{t}_{i}\right) X^{H}\left(\mathrm{t}_{i}\right)$ is the sample covariance matrix, and $H$ denotes the Hermitian conjugate. In this paper, we use the proposed neural network algorithm as the optimization tool, searching for the global optimal solution.

\section{NEURAL NETWORK ALGORITHMS}

The neural network is a kind of Artificial Intelligence which is a good tool to solve non-linear problem in many applications. The neural network filter consists of a feedforward neural network with two or more layers. Neurons, which are computation element in neural network, in any layer, are connected only to neurons in the next layer. The input to the neural network consists of raw signal target values. We use the neural network like a moving window trans form which is ordinary in space time signal processing technique. Operation of the neural network filter over a return signal is similar to the operation of a spatial domain filter. The neural network as a filter has recently been applied to scene segmentation and wafer inspection, but has not been applied in the DOA estimation domain. The neural network filter is convolved with the signal returning to produce output at each detector. The neuron output is scaled across the threshold level range. The neural network filtering thus produces a decision level response map filter. The filter response is supposed to be high for target pixels and low for background pixels. The filtered image can be threshold to obtain the intermediate object location map. False alarm rates can be controlled by threshold selection strategies, low thresholds being favored at the detector stage so as not to preclude any targets from subsequent stages. The requirements for the detection stage are a high detection rate with a low false alarm rate.

The Hopfield model neural network is a single layer of fully inter-connected neurons that update their outputs upon sampling the outputs of other neurons in the network, via the synaptic link.

The synaptic link between the ith and the jth neurons, in a network of $\mathrm{P}$ neurons, form a symmetric matrix $\mathrm{T}$ which elements obey the following formula:

$t_{i j}=-t_{j i} ; \quad t_{i i}=1$

The network changes are stated using the following dynamic equation:

$C_{i} \frac{d u_{i}}{d t}=\sum_{j}^{k} k_{i j} w_{j}+N_{i}$

Where Matrix $C$ is the input capacitance of the ith neuron, it is the external input, and is the internal state of the neuron. The output state $V i$ of the neuron is given by the following nonlinear transformation:

$$
\begin{aligned}
& v_{i}=g_{i}=\frac{1}{1+e^{-\frac{v_{i}}{\eta}}} \\
& \text { for } v_{i} \in\{-1,+1\}
\end{aligned}
$$

where $g_{i}$ is the sigmoid transfer function of the ith neuron, and $1 / \eta$ is the gain of the neuron. The network dynamic equation defines a complex system, but it is possible to find an energy function satisfying the Lyapunov's stability Criterion

$$
E=-\frac{1}{2} \sum \sum w_{i j} v_{i} v_{j}-\sum v_{i} I_{i}
$$

Under above conditions, the Hopfield model implements a gradient descent algorithm, and given the complex multimodal cost function of the feature extra problem, the network is liable to find a local rather than the global minimum. To overcome this problem and increase the probability of finding the global minimum, some modifications have been proposed. 
The decision space of a $P$-neuron network is represented by a $P$-dimensional hypercube $D\left([0,1]^{p}\right)$. Each corner of this hypercube represents a possible digit output state of the network; one of these corners represents the solution state, and one or more of the other corners represents the local-minima of the energy function. The network starts from some initial state within $D\left([0,1]^{p}\right)$ and develops toward one of the corners that corresponds to minimum.

For a digit neuronal trader function, the global minimum must be one of the comers of the hypercube. However for an analog network with a sigmoid transfer function, due to the reason of an analog transfer function represent a perturbation to the energy function, the global minimum must not be one corner of the hypercube.

Yet provided that the neuronal gain is high enough to eliminate the perturbation of the digit energy function, the analog network can be replaced by digital network.

Therefore the global minimum may be thought to be one of the corners of hypercube. We can calculate the energy of all $\mathrm{m}$ e $\mathrm{m}$ on the hypercube directly so long as the $P$ is not very large. Then after the computation of the energy of all formula, the $P$ is the global minimum which we need. Here we call it Energy Comparing method (ECM). The advantage of ECM that any other modification doesn't pose that is it ultimates the local minimum completely. And the computational complexity does not increase obviously so long as the number of neurons is not very large.

For the pulse radar, first suppose that the target moves so slowly that the return sequences of the target in the $H$ times Pulse repetition interval $P R I$ are at the same position. The changing pattern of the return sequences of the target will manifest some specific distributions in the amplitude and frequency feature spaces. Furthermore, the distribution will possess considerable stability in a distant range.

Suppose is the column vector composed of the $t_{t h}$ sampling point in the each return sequence.

\section{THE NN-BASED ML ESTIMATION}

The effectiveness of DOA estimation is guaranteed when the network is solved with the target echo. Since the output of the network is zero and one, the features may be coded by the natural order of the output of the Hopfield networks. Of course, the best way of estimation is that the distinct feature will be shown clearly after coding. When we do not have any prior knowledge about the feature distributions, we may use the above coding method.

The course of synthesizing two kinds of distinct features is, in fact, a course of features integration which transforms distinct input feature spaces into the same output space so as to make detection and decision.

Consider the simple case of a continuous function mapping from a 2-dimensional $\mathrm{z}, \mathrm{y}$ input space to a 1dimensional $\mathrm{z}$ output space. It is theoretically possible to model this mapping with a number of 2-dimensional radial functions. A radial function is one whose evaluation depends upon a radial distance from the function center.
Our networks use Gaussian radial functions which, in 2dimensions, look like bumps or hills. The Gaussian bumps are placed in a flat $\mathrm{z}=0$ function space at input locations where the desired mapping output is known, i.e. training points. The bumps mold the space to the correct answer for the training samples and distort the space around the training point in a continuous fashion. Multiple bumps overlap to model complex areas. The bumps may change their location, width, and height during training to encompass additional samples. Theoretically, this method can approximate any continuous function to any degree of accuracy. The challenge lies in selecting an appropriate set of training samples to place sufficient bumps in the function space to model the function to the desired accuracy. A neural network for an element antenna uses an input space with 14 dimensions (the sine and cosine of 7 phase differences). The inputs are mapped to an output space of "energy bins" representing azimuth angles off antenna broadside. Energy in the bins represents areas with targets.

A DOA estimation network consists of three layers of nodes, input, Gaussian, and output, which are fullyconnected by two layers of arcs, center and weight. "Fullyconnected" means all nodes in layer i are connected to all nodes in layer $i+1$. There are no connections between nodes in the same layer. The function space is primarily shaped by the Gaussian nodes.

The input nodes, I, in the DOA estimation network accept preprocessed input data and fan it out to the center arcs. The center arcs connect each input to each Gaussian node and calculate the distance for that dimension from the current input value to a given Gaussian, $\mathrm{k}$, i.e.

$C_{i j}=I_{j}-m_{j k}$

where $I$, is the $j_{t h}$ input dimension and $m_{j k}$ is the center of the $k_{t h}$ Gaussian node in the $k_{t h}$ input dimension. If the distance from center to input in each dimension is small or zero, that Gaussian is "relevant" to the answer, i.e. it's center in the function space is near the current input.

In ML estimation problem, we want to maximize the function $L(\Theta)$ in (6). According to $\mathrm{NN}$ algorithm, we first initialize some parameters, then a set of data is employed to train the neural network to get the neuron parameters. At last, when new signal data is arriving, they are processed by the trained neural network to estimate the direction-of-arriving of the target.

\section{SIMULATIONS}

In order to verify the performance of the ML estimator computed by our proposed neural network algorithm, we compared it with the alternating projection method proposed by Ziskind and Wax [6].

In the simulations, the array is linear and uniform with three isotropic sensors spaced half a wavelength apart. The sources are two equal power narrow-band emitters, and the noise is additive and uncorrelated from sensor to sensor and with the signals. 


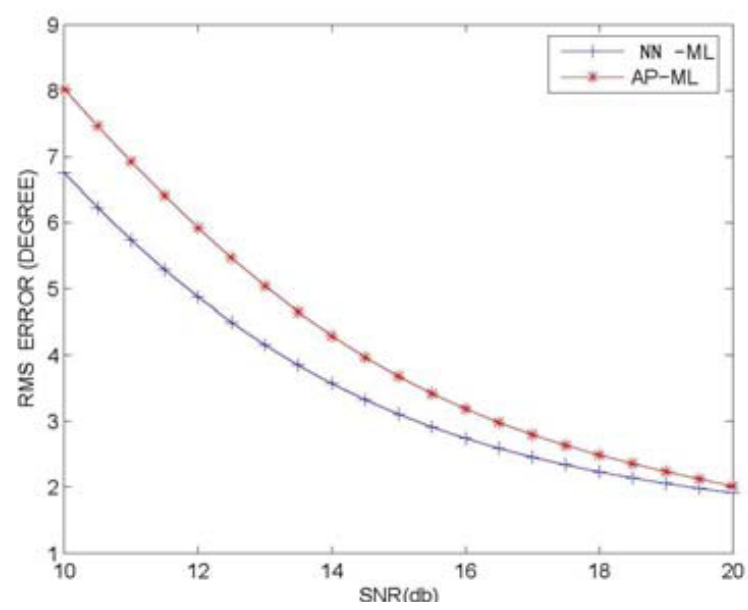

Fig. (1). The resulted rms error (in degrees) of the first source as a function of the SNR.

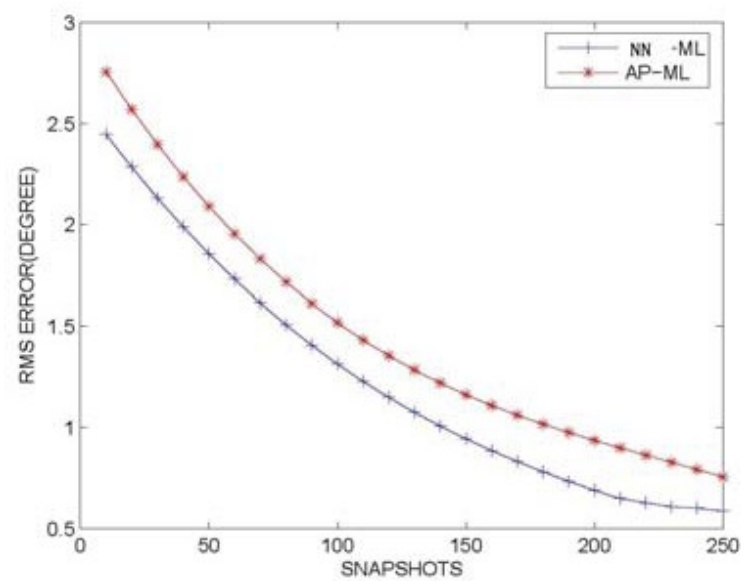

Fig. (2). The resulted rms error of the first source as a function of the number of snapshots.

In every experiment we perform 100 Monte-Carlo runs and compute the root-mean-square (rms) error for each direction-of-arrival.

In the first simulation we simulated two uncorrelated emitters impinging between $0^{\circ}$ and $20^{\circ}$. The number of snapshots taken is 20. Fig. (1) shows the resulted rms error (in degrees) of the first source as a function of the SNR, defined as $\mathrm{SNR}=10 \log \left(\mathrm{s}^{2} / \sigma^{2}\right)$ (where $\mathrm{s}^{2}$ and $\sigma^{2}$ are the average power of the signals and the noise, respectively). The improved performance of the NN based ML estimator at low and moderate SNR is evident.

In the second experiment, the scenario is the same as in the first one, except that this time we fix the SNR to $20 \mathrm{~dB}$. Fig. (2) shows the resulted rms error of the first source as a function of the number of snapshots.

From the experiment, we can see NN algorithm outperforms AP algorithm. Moreover, in our experiment, AP algorithm sometimes does converge to a local optimum. And we solved it successfully by NN algorithms.

\section{CONCLUSION}

In this paper, we have proposed a new algorithm for computing the ML estimator of the direction of multiple sources in the far field by using NN technique. The algorithm is iterative. This is conspicuous advantage over traditional algorithm.

\section{CONFLICT OF INTEREST}

The authors confirm that this article content has no conflict of interest.

\section{ACKNOWLEDGEMENTS}

Declared none.

\section{REFRENCES}

[1] F. C. Schweppe, "Sensor array data processing for multiple signal sources," IEEE Trans. Inform. Theory, vol. 14, no. 3, pp. 294-305, 1968.

[2] R. O. Schmidt, "Multiple emitter location and signal parameter estimation," IEEE Trans. Antennas Propagat., vol. 34, no. 3, pp. 276-280, 1986.

[3] S. S. Reddi, "Multiple source location- A digital approach," IEEE Trans. Aerosp. Electron. Syst., vol. AES-15, pp. 95-105, 1979.

[4] R. Kumaresan and D.W. Tufts, "Estimating the angles of arrival of multiple plane waves," IEEE Trans. Aerosp. Electron. Syst., vol. AES-19, pp. 134-139, 1983. 
[5] L.C. Godara, "Application of antenna array to mobile communications, part ii: beam-forming and direction-of- arrival considerations" In: Proc. IEEE, vol. 85, pp. 1195-1245, 1997

[6] I. Ziskind and M. Wax, "Maximum likelihood localization of multiple sources by alternating projection," IEEE Trans. Acoust., Speech Signal Proc., vol. 36, no. 10, pp. 1553-1560, 1988

[7] K. Sharman, "Maximum likelihood estimation by simulated annealing", Proc. ICASSP'88, New York, 1988, pp. 2741-2744.
[8] P. Stoica and A.B. Gershaman, "Maximum- likelihood DOA estimation by data-supported grid search", IEEE Signal Proces. Lett., vol. 6, pp. 273-275, 1999

[9] P. Stoica and K. C. Sharman, "Maximum likelihood methods for direction-of-arrival estimation", IEEE Trans. Acoust., Speech, Signal Proces., vol. 38, pp. 1132-1143, 1990

[10] F. Obernosterer and W.F. Oehme, "Application of a neural network for detection at strong nonlinear intersymbol interference", IEEE Trans. Magnet., vol. 33, no. 5, pp. 2974-2976, 1997.

Received: July 16, 2014

(C) Zhi-Ming et al.; Licensee Bentham Open.

This is an open access article licensed under the terms of the Creative Commons Attribution Non-Commercial License (http://creativecommons.org/licenses/by$\mathrm{nc} / 4.0 /$ ) which permits unrestricted, non-commercial use, distribution and reproduction in any medium, provided the work is properly cited. 